\title{
Dermatologic Manifestations, Histologic Features and Disease Progression among Cutaneous Lupus Erythematosus Subtypes: A Prospective Observational Study in Asians
}

Kumutnart Chanprapaph · Jutamas Tankunakorn · Poonkiat Suchonwanit •

Suthinee Rutnin (D)

Received: October 22, 2020 / Accepted: November 21, 2020 / Published online: December 5, 2020

(c) The Author(s) 2020

\section{ABSTRACT}

Introduction: Cutaneous manifestations are central to the primary diagnosis of systemic lupus erythematosus (SLE). However, information on the clinical, histopathologic, and direct immunofluorescence (DIF) features among subtypes of cutaneous lupus erythematosus (CLE), as well as longitudinal prospective observational study to evaluate the natural history and the progression to SLE, is lacking among Asians. Our objectives are to summarize the differences in the clinical, histopathologic, and DIF characteristics and serological profiles between various subtypes of CLE, and to provide its natural history and the association with disease activity in our Asian population.

Methods: A prospective observational study on CLE patients was performed between May 2016 and May 2020. Patients underwent full physical/dermatologic examination, skin biopsy for histology, and DIF. Systemic Lupus Erythematosus Disease Activity Index 2000 (SLEDAI-

Supplementary Information The online version contains supplementary material available at https:// doi.org/10.1007/s13555-020-00471-y.

K. Chanprapaph $\cdot$ J. Tankunakorn .

P. Suchonwanit · S. Rutnin ( $\varangle)$

Division of Dermatology, Faculty of Medicine,

Ramathibodi Hospital, Mahidol University,

Bangkok, Thailand

e-mail: suthinee.rutnin@gmail.com
2K) scores and laboratory data were evaluated. Time schedule and characteristics for resolution and/or the disease progression to SLE were recorded in subsequent follow-ups.

Results: Of 101 biopsy-proven CLE patients, 25 had acute CLE (ACLE), 8 had subacute CLE (SCLE), 39 had chronic CLE (CCLE) only, 22 had CCLE with SLE, and 7 had LE-nonspecific cutaneous lesions only. Patients with exclusive CLE showed lower female preponderance, serological abnormalities, and correlation to systemic disease. However, when CLE was accompanied with any LE-nonspecific cutaneous manifestations, they were associated with high antinuclear antibody (ANA) titer, renal, hematologic, joint involvement, and greater SLEDAI score. Of 207 biopsy sections, SCLE/CCLE regardless of systemic involvement showed significantly higher percentage of superficial/deep perivascular and perieccrine infiltration than ACLE. On DIF, deposition of multiple immunoreactants was associated with higher systemic disease. Approximately $10 \%$ of CLE-only patients later developed SLE but had mild systemic involvement.

Conclusion: Our findings support that each CLE subtype has a diverse and unique character. Comprehensive understanding of the differences among CLE subtypes is important for achieving the correct diagnosis and providing appropriate disease monitoring and management. 
Keywords: Asian; Cutaneous lupus erythematosus (CLE); Systemic lupus erythematosus (SLE); Histopathology; Direct immunofluorescence; LE-associated serology profiles; Systemic Lupus Erythematosus Disease Activity Index 2000 (SLEDAI-2K)

\section{Key Summary Points}

The correlation between clinical, histopathology, immunofluorescence, and serologic profiles remains crucial for accurate diagnosis of cutaneous lupus erythematosus (CLE).

It is important to examine for lupus erythematosus (LE)-nonspecific cutaneous manifestations in CLE patients, as they are indicators of internal lupus with high disease activity.

Histopathologic studies showed distinctive features in different CLE subtypes. On immunopathology, multiple immunoreactant staining on DIF indicated systemic disease.

CLE to systemic lupus erythematous transformation occurred in approximately $10 \%$ of chronic CLE-only patients, but the disease remained mild.

\section{DIGITAL FEATURES}

This article is published with digital features to facilitate understanding of the article. You can access the digital features on the article's associated Figshare page. To view digital features for this article go to https://doi.org/10.6084/m9. figshare.13252160.

\section{INTRODUCTION}

Systemic lupus erythematosus (SLE) is a chronic autoimmune inflammatory disease with a wide spectrum of presenting symptoms from localized cutaneous lupus erythematosus (CLE) to severe, life-threatening systemic form [1]. The overall incidences of SLE and CLE are similar: approximately $1-10$ per 100,000 and $2-4$ per 100,000, for SLE and CLE, respectively [2]. Dermatologic manifestations are very common and included in various classification criteria for SLE [3-5]. Skin involvement can be found in $72-85 \%$ of patients and presents as the first clinical sign in one-fourth [6]. Studies have shown that $25 \%$ of patients with newly diagnosed CLE already have a SLE diagnosis, and a subset of these patients eventually develop SLE later $[7,8]$. Durosaro et al. reported a $5 \%$ and $23 \%$ cumulative incidence of SLE in newly diagnosed CLE at 5 and 25 years, respectively [9].

Lupus erythematous (LE) associated skin findings have varied expression, leading to a broad classification as LE-specific and LE-nonspecific skin diseases based on dermatologic features and histological analysis [10-12]. LEspecific skin disease refers to CLE with one or more of the following histologic features: interface changes with basal vacuolization, hyperkeratosis, thickening of the epidermal basement membrane, perivascular/perifollicular mononuclear cell infiltrate, and dermal mucin [11]. LE-nonspecific skin signs refer to cutaneous findings that, although driven by underlying SLE, do not possess the aforementioned histologic features of CLE. LE-specific skin signs include acute CLE (ACLE), subacute CLE (SCLE), and chronic CLE (CCLE). LE-nonspecific skin manifestations comprise of mucosal ulcer, nonscarring alopecia, cutaneous vasculitis, Raynaud's phenomenon, etc. [10-12]. The identification of LE-nonspecific but disease-related skin lesions is important, as their presence may indicate systemic disease.

The diagnosis of LE-associated skin signs is traditionally based on the correlation of clinical findings, histopathology, and serology. Classifying LE-related skin findings into different subsets and understanding its natural history are important to distinguish the clinical presentation and consequences for optimal treatment and appropriate patient monitoring. However, a prospective longitudinal study to elaborate the true natural history of each CLE 
subtype is limited. Moreover, investigations of CLE from a dermatologist's standpoint are relatively sparse compared with SLE from a rheumatologist's standpoint. In addition, reports on Asian CLE compared with nonAsians remains minimal and confined to a study from Japan, Singapore, and Korea [13-15]. Therefore, the aim of this study was to describe the clinical, histologic, and immunopathologic features and LE-associated serological profiles in Thai CLE patients. We also analyzed the differences among CLE subtypes in relations to SLE disease severity. Finally, we prospectively explored the natural history of each CLE subtype and the progression to SLE in a 3-year follow-up period.

\section{METHODS}

This prospective observational study was approved by the Mahidol University Institutional Review Board for Ethics in Human Research (MURA2560/266). All procedures performed involving human participants were in accordance with the institutional research committee and with the 1964 Helsinki Declaration and its later amendments or comparable ethical standards. Informed consent to participate in the study and for the publication of this article was obtained from all individual participants included in the study.

We recruited clinically compatible CLE patients confirmed by two certified dermatologists and one certified dermatopathologist from the dermatology outpatient and inpatient department at Ramathibodi Hospital, Bangkok, Thailand. All patients had a minimum followup period of 3 years. The exclusion criteria included patients with overlap connective tissue diseases at first diagnosis, patients with only one visit, and patients lost to follow-up in less than 6 months.

\section{Demographic, Clinical, and Laboratory Evaluations}

The following variables were involved in the descriptive analysis: sex, age at initial diagnosis, duration of disease, lesion morphology, site of involvement, and systemic manifestations. CLE was defined to LE-specific and LE-nonspecific cutaneous manifestations based on clinical and histopathological findings [10]. Laboratory findings included complete blood count (CBC), urinalysis (UA), urine protein/creatinine ratio (UPCR), erythrocyte sedimentation rate (ESR), and serum complement levels (C3, C4, CH 50). Serologic screening for lupus included antinuclear antibody (ANA) and anti-double stranded DNA antibodies (anti-dsDNA). ANA titer of $\geq 1: 80$ was defined as positive, $1: 320$ and $1: 640$ were considered as moderated titer, and $\geq 1: 1280$ as high titer [16]. Antibodies for extractable nuclear antigens (ENA) were determined by using a commercial enzyme immunological method and included the following antibodies: anti-Ro/SSA, anti La/SSB antibodies, anti-nRNP/sm antibody, anti-Smith (anti-Sm) antibodies, anti-U1-ribonucleoprotein/ribosomal-P-proteins (anti-U1-RNP) antibodies, anti-histone antibodies, anti-Scl-70 antibodies, anti-centromere antibodies (antiCENP-B), and anti-Jo-1 antibodies. Patients with more than one CLE subtype were classified according to all appropriate subtypes to analyze on the clinical characteristics, histopathology, and DIF. However, with regards to the serological abnormalities and disease severity, patients with more than one CLE type were categorized into subgroups with the highest risk of systemic involvement; For example, patients with clinical photosensitivity and malar rash with isolated scarring DLE lesions were classified as having ACLE.

\section{Histopathologic and Immunofluorescence Study}

Patients underwent two skin biopsies for histological and DIF using a 4-mm punch biopsy instrument. The first section was stained with hematoxylin-eosin and assessed for pathologic changes reported by a dermatopathologist. The histopathological characteristics were reviewed from the epidermis down to subcutaneous tissue. The following features were evaluated: epidermal changes (normal, atrophy/thinning, hyperplasia, hyperkeratosis, dilated follicle with 
follicular plugging), interface change (basal vacuolization, lichenoid infiltrate), pattern of dermal cell infiltrate (superficial perivascular infiltrate, superficial and deep perivascular infiltrate, periadnexal infiltrate), inflammatory patterns of the subcutaneous tissue (septal panniculitis, lobular panniculitis, mixed septal and lobular panniculitis), dermal and subcutaneous cytologic composition (lymphocytes, neutrophils, eosinophils, plasma cells, macrophages, granuloma, extravasated erythrocytes), vasculitis, dermal fibrosis, mucin deposition, and fat necrosis (hyalinized sclerosis, lipomembranous fat necrosis). Variable degrees of these features were then classified into ACLE, SCLE, and CCLE. The other biopsy specimen for DIF was processed and stained to detect the presence of bound immunoglobulin (IgG, IgM, IgA) and complement (C3) reported by an immunodermatologist.

\section{Defining Progression to SLE}

A patient was considered to have experienced a transition to SLE if they had a CLE diagnosis at first visit and went on to fulfill the Systemic Lupus International Collaborating Clinics (SLICC) criteria [3] during the follow-up period.

\section{Classifying Severity}

We determined the severity of all systemic symptoms present at first visit by using the Systemic Lupus Erythematosus Disease Activity Index 2000 (SLEDAI-2K) [17]. Total SLEDAI-2K score falls between 0 and 105, with higher scores representing higher disease activity. The British Isles Lupus Assessment Group's activity index (BILAG2004) [18], a more reliable tool than the SLEDAI-2K in monitoring disease activity with treatment, was used to determine systemic involvement in CLE patients who progressed to SLE. The score evaluated nine system involvement and disease activity which is later established into five levels: categories $\mathrm{A}-\mathrm{E}$, with $\mathrm{A}$ indicating very active disease and $\mathrm{E}$ representing no current or previous disease activity [19].

\section{Statistical Analysis}

Statistical analysis was completed on Stata 14.0 (StataCorp LLC, College Station, TX, USA). To create an overall picture of the differences among the lupus subtypes, several types of comparisons were made; group frequencies or categorical traits were compared with a chisquared test or Fisher's exact test. Differences in SLEDAI-2K for each CLE variable were calculated using Mann-Whitney $U$-test. The Kruskal-Wallis tests was used for comparisons between more than two independent groups. $p$ Value less than 0.05 was considered significant.

\section{RESULTS}

Of 109 patients, 5 were excluding due to overlapping diagnosis with other connective tissue diseases, 2 had less than 6 months follow-up, and 1 had follow-up on only one visit. Of the remaining 101 patients, 59 had underlying SLE and 42 were diagnosed with CLE only. A total of 190 lesions were divided into LE-specific $(n=117,61.58 \%)$ and LE-nonspecific $(n=73$, $38.42 \%$ ) cutaneous lesions. Details regarding study protocol are provided in Fig. 1.

Of 101 biopsy-proven CLE patients, 7 had exclusive LE-nonspecific skin manifestation. The remaining 94 patients were then divided into four subgroups: 25 with ACLE (24.8\%), 8 with SCLE (7.9\%), 39 with CCLE-only (38.6\%), and 22 with CCLE and SLE (21.8\%). A total of 11 patients were diagnosed with more than one CLE subtypes: ACLE combined with CCLE $(n=9)$, SCLE combined with CCLE $(n=1)$, and all three CLE subtypes $(n=1)$. Mean age at diagnosis was not significantly different between subgroups $(p=0.09)$. Female predominance was apparent in all groups. A significant reduction of male-to-female ratio was observed in patients with CCLE-only (F: $\mathrm{M}=2.25: 1$ ) compared with ACLE (24:1), SCLE (7:1), and CCLE with SLE (10:1) $(p=0.027)$. Table 1 summarizes the patients' demographic data.

There were 117 LE-specific cutaneous lesions in 94 patients. The most common LE specific cutaneous lesion was CCLE $(n=75,64.1 \%)$, followed by ACLE $(n=34,29.1 \%)$ and SCLE 


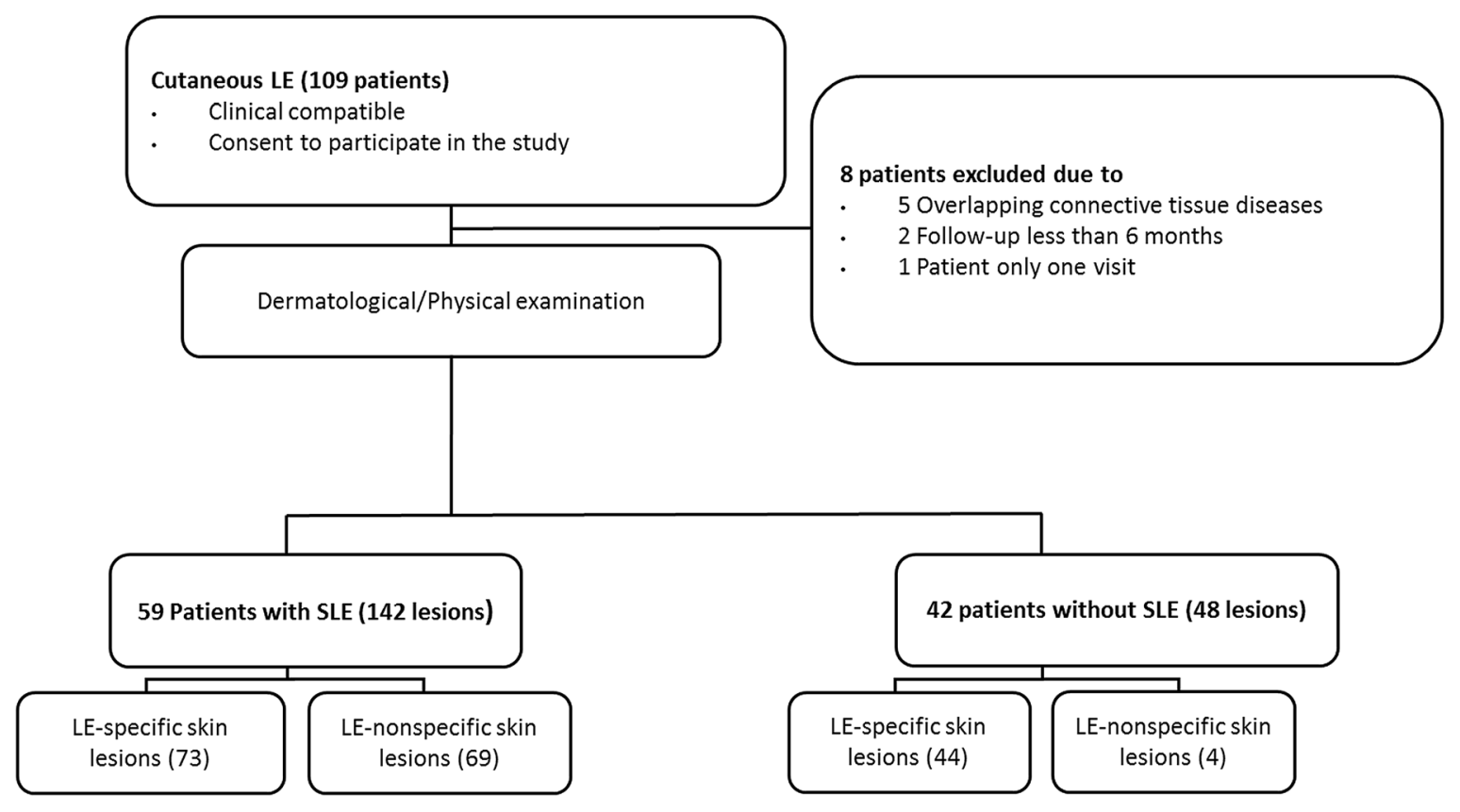

\section{LE, lupus erythematosus; SLE, systemic lupus erythematosus}

Fig. 1 Flowchart describing participant selection

Table 1 Demographic and clinical characteristics of cutaneous lupus erythematosus patients

\begin{tabular}{|c|c|c|c|c|c|}
\hline \multirow[t]{2}{*}{ Demographic characteristics } & \multicolumn{4}{|c|}{ Cutaneous lupus erythematosus, $n$ (\%) } & \multirow{2}{*}{$p$-value } \\
\hline & $\begin{array}{l}\text { ACLE } \\
n=25(24.8)\end{array}$ & $\begin{array}{l}\text { SCLE } \\
n=8(7.9)\end{array}$ & $\begin{array}{l}\text { CCLE-only } \\
n=39(38.6)\end{array}$ & $\begin{array}{l}\text { CCLE + SLE } \\
n=22(21.8)\end{array}$ & \\
\hline Mean age, year $\pm S D$ & $38.2 \pm 17.7$ & $51.0 \pm 24.4$ & $44.8 \pm 15.6$ & $51.0 \pm 21.8$ & 0.090 \\
\hline \multicolumn{6}{|l|}{ Sex } \\
\hline Male & $1(4)$ & $1(12.5)$ & $12(30.8)$ & $2(9.1)$ & \\
\hline Female & $24(96)$ & $7(87.5)$ & $27(69.2)$ & $20(90.9)$ & \\
\hline Female: Male & $24: 1$ & $7: 1$ & $2.25: 1$ & 10:1 & 0.027 \\
\hline Southeast Asian ethnicity & $25(100)$ & $8(100)$ & $39(100)$ & $22(100)$ & \\
\hline Time to CLE diagnosed; days (IQR) & $16.9(7-30)$ & $\begin{array}{l}111.3 \\
\quad(52.5-105)\end{array}$ & $\begin{array}{l}553.4 \\
\quad(30-730)\end{array}$ & $398.8(30-365)$ & $<0.001$ \\
\hline $\begin{array}{l}\text { Median duration of follow-up; days } \\
\text { (IQR) }\end{array}$ & $\begin{array}{l}878 \\
\quad(525-1346)\end{array}$ & $\begin{array}{l}664 \\
\quad(267-1060)\end{array}$ & $\begin{array}{l}792 \\
(469-1099)\end{array}$ & $\begin{array}{l}822 \\
\quad(425-1179)\end{array}$ & 0.606 \\
\hline Time to resolution; days (IQR) & $24.5(7-31)$ & $49(22-47)$ & $140(45-128)$ & $81(24-106)$ & 0.001 \\
\hline
\end{tabular}

Bold indicates statistically significant

$A C L E$ acute cutaneous lupus erythematosus, $C C L E$ chronic cutaneous lupus erythematosus, IQR interquartile range, $L E$ lupus erythematosus, $S C L E$ subacute cutaneous lupus erythematosus, SLEDAI-2K Systemic Lupus Erythematosus Disease Activity Index 2000, year $\pm S D$, year \pm standard deviation 
$(n=8,6.8 \%)$. Almost half of ACLE lesions were localized ACLE $(n=14,41.2 \%)$, characterized by symmetrical fixed erythematous patches appearing on both cheeks and nose, and sparing the nasolabial folds (Fig. 2a). Generalized ACLE presented as widespread morbilliform eruption $(n=9,26.5 \%)$ and photosensitivity rash $(n=7$, 20.6\%). A few patients experienced Stevens-Johnson syndrome/toxic epidermal necrolysis (SJS/TEN)-like ACLE $(n=3,8.8 \%)$. Finally, the rarest form of ACLE was erythema multiforme (EM)-like ACLE $(n=1,2.9 \%)$. The median resolution time for ACLE was 24.5 (IQR 7-31) days. The majority of ACLE lesions resolved with post-inflammatory hypo- and/or hyperpigmentation ( $n=27,79.4 \%$ ) (Fig. 2b).

SCLE lesions presented initially as erythematous macules or papules that evolved into scaly papulosquamous $(n=7,87.5 \%)$ or annular/polycyclic plaques $(n=1,12.5 \%)$ (Fig. $2 \mathrm{c}$ ). Approximately one-third of SCLE cases had no systemic involvement. The majority of SCLE lesions were on sun-exposed areas, e.g., dorsal aspects of the arms/forearms $(n=4,50 \%)$, upper back $(n=3,37.5 \%)$ and posterior neck $(n=1,12.5 \%)$. The median healing time for SCLE was 51.4 days (IQR 16-90 days). All SCLE lesions subsided without scaring, but resulted in post-inflammatory erythema $(n=4,50 \%)$ (Fig. 2d), post-inflammatory hypopigmentation ( $n=2,25 \%)$, post-inflammatory erythema with telangiectasia ( $n=1,12.5 \%)$, and post-inflammatory hyperpigmentation $(n=1,12.5 \%)$.

The most common form of CCLE was classic DLE $(n=42,56 \%)$, presenting with red macules, papules, or small plaques and adherent follicular hyperkeratosis. DLE lesions occurred most frequently on the head and neck $(n=24$, $57.1 \%$ ) (Fig. 2e), followed by the upper extremities $(n=13,31 \%)$ and trunk $(n=5$, $11.9 \%)$. The majority of DLE lesions evolved into central atrophic scars with peripheral hyperpigmentation ( $n=38,90.4 \%)$ (Fig. 2f), followed by post-inflammatory hypo- or hyperpigmentation $(n=2,4.8 \%)$ and post-inflammatory erythema $(n=2,4.8 \%)$. Lupus panniculitis was diagnosed in approximately one-fifth of CCLE ( $n=16,21.3 \%)$, and half had overlying DLE lesions $(n=8,50 \%)$. One patient $(n=1,6.3 \%)$ presenting with periorbital edema.
The most common distributions for LE panniculitis were the head and neck $(n=8,50 \%)$, followed by the trunk $(n=7,43.8 \%)$ and upper extremities $(n=1,6.2 \%)$. Lesions of LE panniculitis eventually subsided in 91.2 days (IQR 54-119 days) leaving residual indentation. Tumid LE $(n=8,10.7 \%)$ presented with annular, indurated, erythematous, edematous plaques predominately located on the head and neck $(n=6,75 \%)$, followed by upper extremities $(n=2,25 \%)$. The median resolution time for tumid LE was 58 days (IQR 30-84 days), and most resolved with post-inflamed erythema $(n=7,87.5 \%)$. Lichenoid DLE $(n=6,8 \%)$ appeared as violaceous papules $(n=3,50 \%)$ or small plaques $(n=3,50 \%)$ with adherent scales on the upper $(n=4,66.7 \%)$ and lower extremities $(n=2,33.3 \%)$. Lichenoid DLE took the longest time to improve with an average resolution time of 120 days (IQR 21-154 days). All lesions healed with brownish atrophic patches and peripheral hyperpigmentation. Finally, the rarest CCLE variant was hypertrophic DLE $(n=3,4 \%)$ which presented with hyperkeratotic verrucous violaceous/brownish plaques on the lower extremities (Fig. 2g). They healed with decreased thickness and post-inflammatory hyperpigmentation within a median time of 68 days (Fig. $2 \mathrm{~h}$ ).

There were 73 LE-nonspecific cutaneous lesions in 39 patients (38.6\%). Among LE-nonspecific skin lesions, non-scarring alopecia was the most common finding $(n=39,53.5 \%)$, followed by cutaneous vasculitis/vasculopathy $(n=20,27.4 \%)$, BSLE $(n=6,8.2 \%)$, Raynaud phenomenon $(n=6,8.2 \%)$, and periungual telangiectasia ( $n=2,2.7 \%)$. A summary of the clinical characteristic of CLE is presented in Table 2.

\section{Histopathology and DIF Assessments}

A total of 207 biopsy specimens were obtained. Overall, 124 lesions underwent histological examination. There were 109 CLE lesions [ACLE $(n=21)$, SCLE $(n=7)$, CCLE $(n=81)]$, and 15 LE-nonspecific skin lesions [BSLE $(n=6)$, nonscaring alopecia $(n=6)$, cutaneous small vessel vasculitis $(n=3)]$. A comparison between 

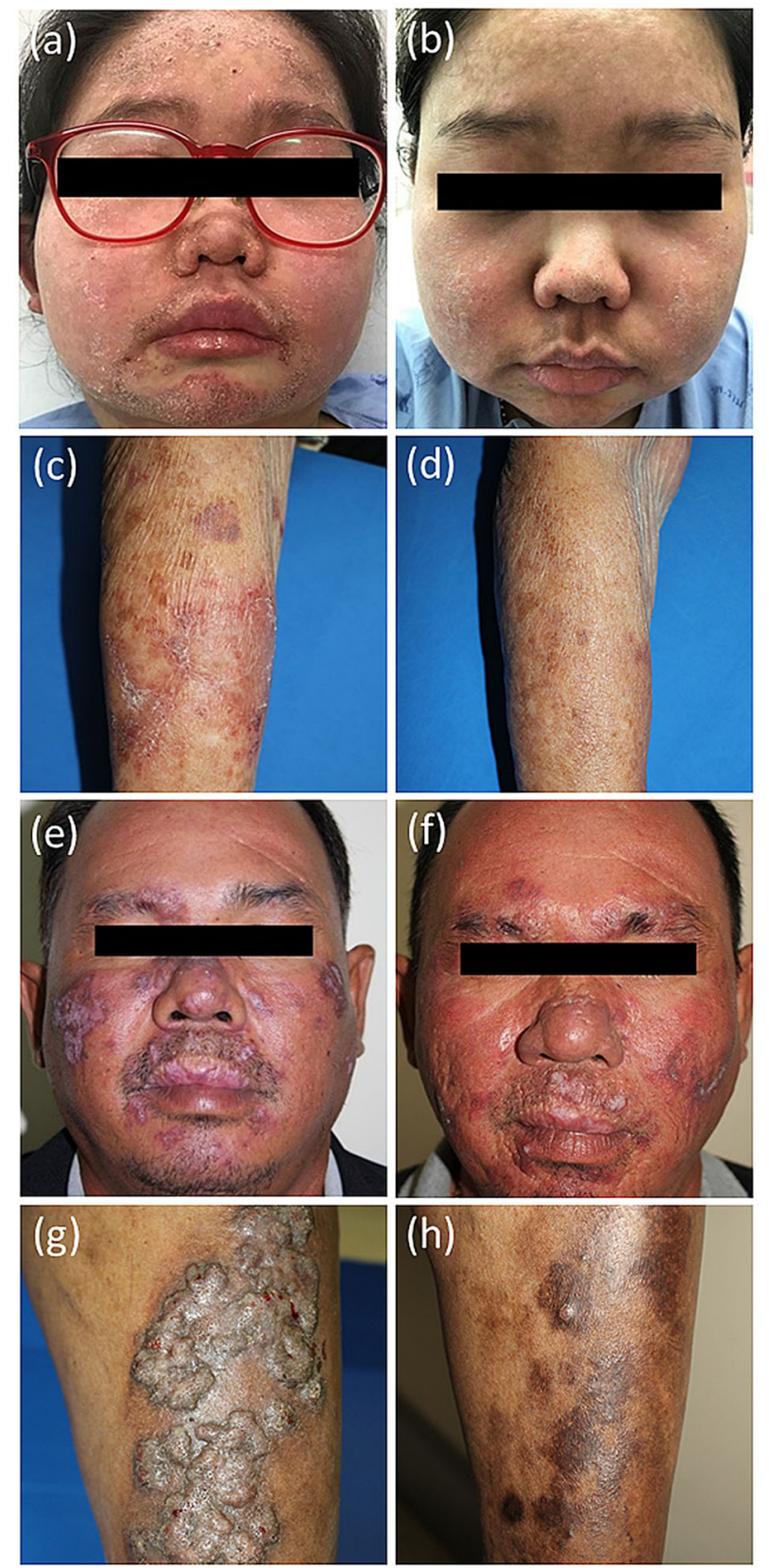

Fig. 2 Acute cutaneous lupus erythematosus (ACLE) a resolving with post-inflammatory hyperpigmentation b in 7 days after treatment with prednisolone, hydroxychloroquine, mycophenolate mofetil, azathioprine, and topical mometasone. Annular subacute CLE c resolving with post-inflammatory erythema $\mathbf{d}$ in 14 days with topical desoximetasone. Discoid lupus erythematosus (DLE) treated with hydroxychloroquine and topical mometasone e that healed with atrophic scar and peripheral hyperpigmentation $\mathbf{f}$ in 49 days. Hypertrophic DLE $\mathbf{g}$ that had gradual improvement after intralesional triamcinolone acetonide $(5 \mathrm{mg} / \mathrm{ml})$ and topical clobetasol with decrease thickness and post-inflammatory hyperpigmentation $\mathbf{h}$ in 68 days 
Table 2 Clinical characteristics of cutaneous lupus erythematosus

\section{CLE with SLE $(n=59)$}

CLE without $\operatorname{SLE}(n=42)$

LE-specific lesions, $n(\%)$

\begin{tabular}{|c|c|c|}
\hline \multicolumn{3}{|l|}{$\operatorname{ACLE}(n=34)$} \\
\hline Lupus malar rash & $14(41.2)$ & 0 \\
\hline Maculopapular lupus rash & $9(26.5)$ & 0 \\
\hline Photosensitive lupus rash & $7(20.6)$ & 0 \\
\hline SJS/TEN-like & $3(8.8)$ & 0 \\
\hline EM-like & $1(2.9)$ & 0 \\
\hline Total & 34 & 0 \\
\hline \multicolumn{3}{|l|}{$\operatorname{SCLE}(n=8)$} \\
\hline Annular & $1(12.5)$ & 0 \\
\hline Papulosquamous & $4(50)$ & $3(37.5)$ \\
\hline Total & 5 & 3 \\
\hline \multicolumn{3}{|l|}{$\operatorname{CCLE}(n=75)$} \\
\hline Localized DLE & $9(12)$ & $15(20)$ \\
\hline Generalized DLE & $15(20.0)$ & $3(4)$ \\
\hline Lichenoid DLE & $3(4.0)$ & $3(4)$ \\
\hline Hypertrophic DLE & 0 & $3(4)$ \\
\hline Lupus panniculitis/profundus & $1(1.3)$ & $15(20)$ \\
\hline Tumid LE & $6(8)$ & $2(2.7)$ \\
\hline Total & 34 & 41 \\
\hline \multicolumn{3}{|l|}{ LE-nonspecific lesions, $n$ (\%) } \\
\hline Non-scarring alopecia & $35(48)$ & $4(5.5)$ \\
\hline Vasculitis/vasculopathy & $20(27.4)$ & 0 \\
\hline Bullous SLE & $6(8.2)$ & 0 \\
\hline Raynaud phenomenon & $6(8.2)$ & 0 \\
\hline Periungual telangiectasia & $2(2.7)$ & 0 \\
\hline Total & 69 & 4 \\
\hline
\end{tabular}

$C C L E$ chronic cutaneous lupus erythematosus, $D L E$ discoid lupus erythematosus, $E M$ erythema multiforme, $L E$ lupus erythematosus, $S L E$ systemic lupus erythematosus, SJS Steven-Johnsons syndrome, $T E N$ toxic epidermal necrolysis

histopathologic findings among CLE subgroups revealed that ACLE had predominant superficial perivascular infiltration $(42.9 \%, \quad p=0.004)$ (Fig. 3a), while SCLE, and CCLE with and without SLE showed superficial/deep perivascular lymphocytic infiltration $(p<0.001)$ and perieccrine infiltration $(p=0.004)$. SCLE sections demonstrated the highest percentage of 

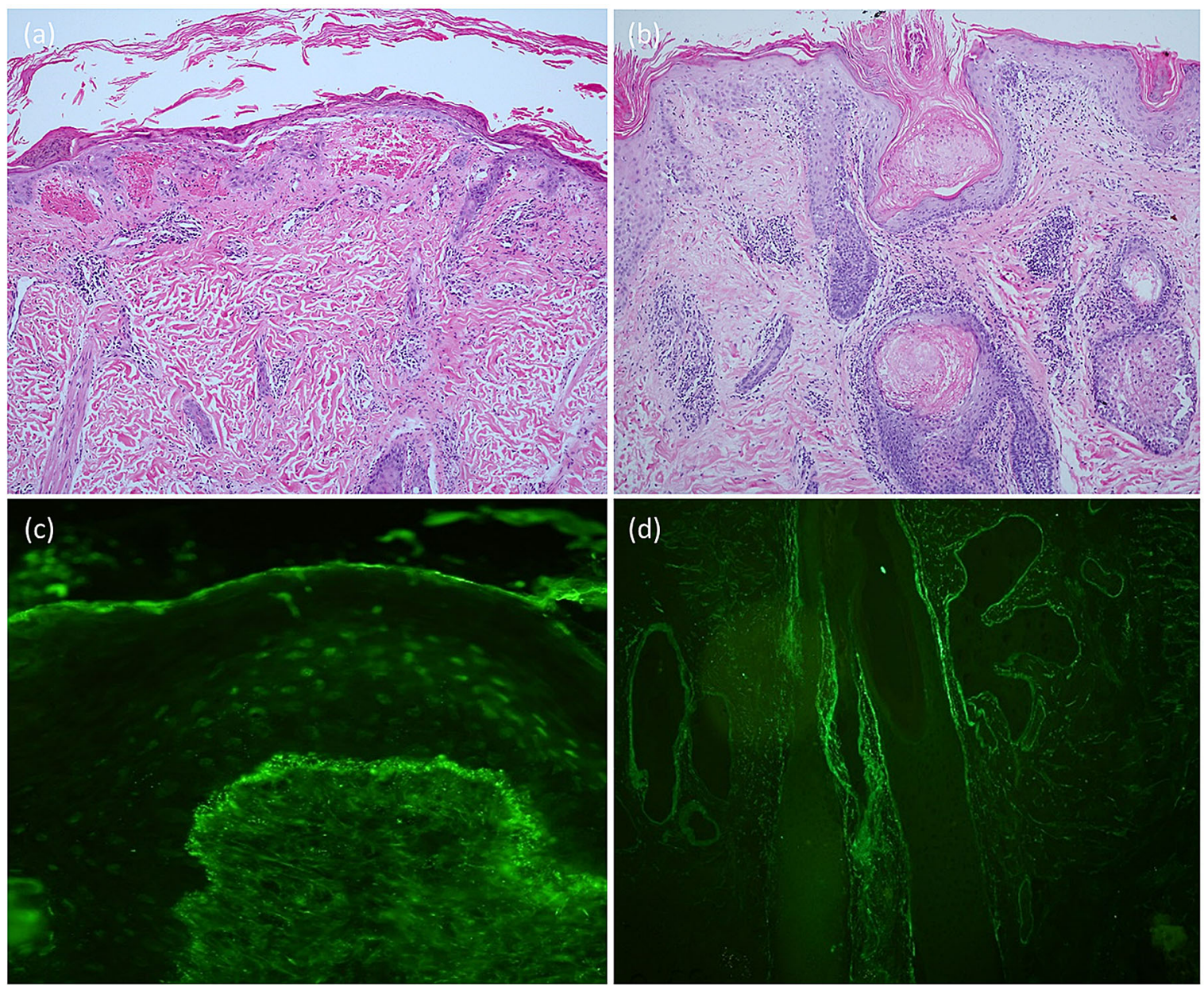

Fig. 3 Histopathological and direct immunofluorescence findings in cutaneous lupus erythematosus. ACLE; superficial perivascular infiltration with lymphocytes and basal vacuolization (a; H\&E, 10×), CCLE; superficial and deep perivascular and perieccrine lymphocytic infiltration (b;

focal hypergranulosis $(25 \%, p=0.036)$. Specimens from CCLE patients had prominent hyperkeratosis with and without follicular plugging (72.4\%, $p=0.004)$ (Fig. 3b). Lobular panniculitis and hyalinized sclerosis were observed exclusively in CCLE lesions and only in lupus panniculitis sections $(p=0.041)$ (Table3).

DIF was examined on 83 CLE sections, of which 13 had confirmation of LE-nonspecific cutaneous lesions, leaving 70 remaining LEspecific skin specimens for analysis. Positive results were demonstrated in 65 CLE sections
H\&E, 10×). ACLE; homogeneous granular deposition along DEJ with epidermal nuclear staining (c), CCLE; deeper perivascular, prominent perifollicular and perieccrine deposition (d)

(92.9\%). Immunoreactants were detected along the dermoepidermal junction (DEJ) on the lesional skin of 63 samples (96.9\%). Patternspecific epidermal nuclear staining corresponded to the serum ANA pattern in ten patients [homogeneous epidermal nuclear staining with homogeneous ANA pattern $(n=6)$, coarse speckle epidermal nuclear staining with speckle pattern ANA pattern $(n=4)]$. The combination of double, triple, or quadruple immunoreactants (IgG, IgA, IgM, and/or C3) was the most frequent staining pattern detected $(89.2 \%)$. CCLE-only was the only subgroup with 
Table 3 Histologic and direct immunofluorescence findings in cutaneous lupus erythematosus

\begin{tabular}{|c|c|c|c|c|c|c|}
\hline \multicolumn{2}{|l|}{ Variable } & $\begin{array}{l}\text { ACLE } \\
(n=21)\end{array}$ & $\begin{array}{l}\text { SCLE } \\
(n=8)\end{array}$ & $\begin{array}{l}\text { CCLE- } \\
\text { only } \\
(n=58)\end{array}$ & $\begin{array}{l}\text { CCLE + SLE } \\
(n=22)\end{array}$ & $p$-value \\
\hline \multicolumn{7}{|l|}{ Histopathologic findings } \\
\hline \multicolumn{7}{|l|}{ Epidermal change } \\
\hline \multicolumn{2}{|l|}{ Epidermal atrophy } & $6(28.6)$ & $5(62.5)$ & $27(46.6)$ & $7(31.8)$ & 0.225 \\
\hline \multicolumn{2}{|l|}{ Necrotic keratinocytes } & $10(47.6)$ & $4(50)$ & $25(43.1)$ & $8(36.4)$ & 0.864 \\
\hline \multicolumn{2}{|l|}{ Focal hypergranulosis } & 0 & $2(25)$ & $4(6.9)$ & 0 & 0.036 \\
\hline \multicolumn{2}{|c|}{ Hyperkeratosis with/without follicular plugging } & $6(28.6)$ & $4(50)$ & $42(72.4)$ & $15(68.1)$ & 0.004 \\
\hline \multicolumn{7}{|l|}{ Interface change } \\
\hline \multicolumn{2}{|l|}{ Basal vacuolization } & $15(71.4)$ & $5(62.5)$ & $36(62.1)$ & $15(68.2)$ & 0.868 \\
\hline \multicolumn{2}{|l|}{ Lichenoid cell infiltration } & $1(4.8)$ & $1(12.5)$ & $6(10.3)$ & $3(13.6)$ & 0.797 \\
\hline \multicolumn{2}{|l|}{ BM membrane thickening } & $1(4.8)$ & $2(25)$ & $10(17.2)$ & $4(18.2)$ & 0.450 \\
\hline \multicolumn{2}{|l|}{ Pigmentary incontinence } & $16(76.2)$ & $5(62.5)$ & $40(69.0)$ & $10(45.5)$ & 0.152 \\
\hline \multicolumn{7}{|l|}{ Dermal change } \\
\hline \multicolumn{2}{|c|}{ Superficial perivascular lymphocytic infiltration } & $9(42.9)$ & 0 & 0 & $4(18.2)$ & 0.004 \\
\hline \multicolumn{2}{|c|}{ Superficial and deep perivascular lymphocytic infiltration } & $6(28.6)$ & $7(87.5)$ & $45(77.6)$ & $17(77.3)$ & $<0.001$ \\
\hline \multicolumn{2}{|l|}{ Perifollicular infiltration } & $3(14.3)$ & $3(37.5)$ & $24(41.4)$ & $9(40.9)$ & 0.153 \\
\hline \multicolumn{2}{|l|}{ Perieccrine infiltration } & $1(4.8)$ & $4(50)$ & $25(43.1)$ & $12(54.6)$ & 0.004 \\
\hline \multicolumn{2}{|l|}{ Increased mucin in dermis } & 0 & 0 & $9(15.5)$ & $1(4.5)$ & 0.098 \\
\hline \multicolumn{2}{|l|}{ Dermal fibrosis } & 0 & 0 & $5(8.6)$ & $1(4.5)$ & 0.422 \\
\hline \multicolumn{7}{|l|}{ Subcutaneous change } \\
\hline \multicolumn{2}{|l|}{ Lobular panniculitis } & 0 & 0 & $9(15.5)$ & $1(4.6)$ & 0.098 \\
\hline \multicolumn{2}{|l|}{ Hyalinized sclerosis } & 0 & 0 & $11(19)$ & $1(4.6)$ & 0.041 \\
\hline & $\begin{array}{l}\text { ACLE } \\
(n=17)\end{array}$ & $\begin{array}{l}\text { SCLE } \\
(n=5)\end{array}$ & \multicolumn{2}{|l|}{$\begin{array}{l}\text { CCLE only } \\
(n=22)\end{array}$} & $\begin{array}{l}\text { CCLE + SLE } \\
(n=21)\end{array}$ & $p$ value \\
\hline \multicolumn{7}{|c|}{ Direct immunofluorescence findings } \\
\hline Epidermal nuclear staining & $8(47.1)$ & $1(20)$ & $3(13.6)$ & & $6(28.6)$ & 0.023 \\
\hline Homogeneous granular DEJ & $15(76.5)$ & $3(20)$ & $15(68.2)$ & & $16(76.2)$ & 0.305 \\
\hline Dermal blood vessels & $6(35.3)$ & $4(80)$ & $9(41)$ & & $9(42.9)$ & 0.047 \\
\hline Perieccrine & $1(5.9)$ & $2(40)$ & $12(54.5)$ & & $5(23.8)$ & 0.009 \\
\hline Perifollicular & $3(17.6)$ & 0 & $6(27.3)$ & & $6(28.6)$ & 0.502 \\
\hline Cytoid bodies & $12(70.6)$ & $1(20)$ & $11(50)$ & & $11(52.4)$ & 0.224 \\
\hline 1 Immunoreactant & 0 & 0 & $7(31.8)$ & & 0 & 0.002 \\
\hline$\geq 2$ immunoreactants & $17(100)$ & $5(100)$ & $15(68.2)$ & & $21(100)$ & 0.002 \\
\hline
\end{tabular}

Bold indicates statistically significant

$A C L E$ acute cutaneous lupus erythematosus, CCLE chronic cutaneous lupus erythematosus, DEJ dermoepidermal junction, SCLE subacute cutaneous lupus erythematosus, $S L E$ systemic lupus erythematosus 
deposition of single immunoreactant on DIF sections $(n=7,31.8 \%, p=0.002)$. The presence of $>1$ immunoreactant was correlated with systemic involvement $(p=0.03)$. A comparison of DIF findings between CLE subtypes showed that ACLE lesions demonstrated the highest percentage of epidermal nuclear staining (47.1\%, $p=0.023$ ) (Fig. 3c, d). SCLE sections showed prominent dermal blood vessels deposition $(80 \%, p=0.047)$. CCLE sections from patients with and without SLE demonstrated the highest percentage of perieccrine infiltration $(54.5 \%, p=0.009)$ (Table3).

There was no statistically significant difference on the following histopathological findings and the corresponding DIF pattern: interface change/basement membrane thickening versus deposition along DEJ $(p=0.95)$, perivascular infiltration versus deposition at dermal blood vessels $(p=0.38)$, periadnexal/ eccrine infiltration and deposition $(p=0.81)$, perifollicular infiltration and deposition $(p=0.30)$, and melanophages/necrotic keratinocytes versus cytoid bodies $(p=0.10)$. However, there was a borderline association between dermal changes on histopathology (perivascular and/or periadnexal infiltration) and DIF (dermal blood vessels and/or periadnexal deposition) $(p=0.069)$.

\section{CLE Subtypes and Laboratory Abnormalities}

CCLE-only patients showed the lowest percentage of moderate to high ANA titer $(\geq 1: 320)$ $(p=0.001)$, anti-DNA $(p=0.001)$, low C3 or C4 level $(p<0.001)$, and mean ESR $(p=0.007)$ compared with other groups. CCLE with SLE patients had higher percentage of moderate to high ANA titer (86.4\%) compared with CCLEonly patients (20.5\%). Among anti-ENAs, only anti-SSA was positively correlated to SCLE $(p=0.004)$ (Table 4).

\section{CLE Subtypes and Disease Activity}

Hematologic, renal, and joint involvement were significantly associated with ACLE, SCLE, and CCLE with SLE more than CCLE-only cases $(p<0.05)$ (Table 4). SLEDAI-2K score (IQR) calculated during the time of examination and skin biopsy was highest in ACLE [10.1 (6-13)], compared with CCLE with SLE [7.2 (4-11)] and SCLE [5.8 (2-8)], respectively. However, there were no statistically significant differences between each group $(p=0.3)$.

\section{CLE Subtypes with and without LE- Nonspecific Cutaneous Manifestations}

Our analysis showed no differences on the disease activity and systemic involvement between ACLE patients with and without LE-nonspecific cutaneous lesions $(p=0.67)$. However, SCLE and/or CCLE patients with any LE-nonspecific skin manifestations (e.g., non-scarring alopecia, vascular reaction, Raynaud's phenomenon, periungual telangiectasia) had significantly higher systemic involvement $(p=0.002)$ and disease activity compared with those without (see Table SI). Of the CCLE-only patients, four patients $(10.3 \%)$ had non-scarring alopecia.

\section{CLE Progression to SLE}

Of 42 CLE-only patients, 4 went on to meet the SLICC criteria for SLE (i.e., 2 generalized DLE, 2 lupus panniculitis). Of note, no exclusive SCLE patients progressed to SLE. All patients had nonscaring alopecia during the course of their disease, and the median progression time was 5.6 (5.1-44.9) months. All had moderate to high initial ANA titer $(\geq 1: 320)$. One generalized DLE and two lupus panniculitis patients later developed mild hematologic and renal diseases (category C). The final case with generalized DLE converted to SLE with only mucocutaneous criteria plus newly established anti-Sm, antiDNA, and hypocomplementemia (category E).

\section{DISCUSSION}

Our prospective observational study confirms the differences in the expression of each CLE subtype with respect to their natural history, histopathology, immunofluorescence, laboratory profiles, and progression to systemic 
Table 4 Autoantibodies, systemic involvement, and systemic lupus diseases activity in cutaneous lupus erythematosus

\begin{tabular}{|c|c|c|c|c|c|}
\hline \multirow[t]{2}{*}{ Variable } & \multicolumn{4}{|c|}{ Patients with LE-specific skin lesions, $n$ (\%) } & \multirow[t]{2}{*}{$p$ value } \\
\hline & $\begin{array}{l}\text { ACLE } \\
n=25(24.8)\end{array}$ & $\begin{array}{l}\text { SCLE } \\
n=8(7.9)\end{array}$ & $\begin{array}{l}\text { CCLE-only } \\
n=39(38.6)\end{array}$ & $\begin{array}{l}\text { CCLE + SLE } \\
n=22(21.8)\end{array}$ & \\
\hline ANA $<1: 320$ & $2(8)$ & $2(25)$ & $28(71.8)$ & $3(13.6)$ & 0.001 \\
\hline ANA $\geq 1: 320$ & $23(92)$ & $6(75)$ & $8(20.5)$ & $19(86.4)$ & \\
\hline Anti-dsDNA antibody & $16(66.7)$ & $3(75)$ & $1(5.0)$ & $7(46.7)$ & 0.001 \\
\hline Anti-Sm antibody & $5(24)$ & $1(16.7)$ & $2(7.7)$ & $6(31.6)$ & 0.125 \\
\hline Anti-SSA autoantibody & $13(61.9)$ & $5(83.3)$ & $5(19.2)$ & $7(36.8)$ & 0.004 \\
\hline Anti-SSB (La) autoantibody & $4(19)$ & $1(16.7)$ & $1(3.8)$ & $4(21.1)$ & 0.179 \\
\hline Anti-nRNP/Sm & $9(42.9)$ & $1(16.7)$ & $7(26.9)$ & $8(42.1)$ & 0.480 \\
\hline $\operatorname{ESR}(\mathrm{mm} / \mathrm{h})(\mathrm{IQR})$ & $48.1(27.68)$ & $29.3(18.32)$ & $24.6(10.44)$ & $41.7(20.62)$ & 0.007 \\
\hline High ESR $(>20 \mathrm{~mm} / \mathrm{h})$ & $22(88)$ & $3(37.5)$ & $17(43.6)$ & $16(72.7)$ & 0.001 \\
\hline Low C3 $(<0.9 \mathrm{~g} / \mathrm{L})$ & $19(79.2)$ & $3(50)$ & $2(9.5)$ & $13(68.4)$ & $<0.001$ \\
\hline Low C4 $(<0.1 \mathrm{~g} / \mathrm{L})$ & $8(33.3)$ & $2(33.3)$ & 0 & $10(50)$ & $<0.001$ \\
\hline \multicolumn{6}{|l|}{ Hematologic involvement } \\
\hline Hemolytic anemia & $2(8)$ & $2(25)$ & 0 & $4(18)$ & 0.008 \\
\hline Leukopenia & $4(16)$ & $2(25)$ & $2(5)$ & $8(36)$ & 0.012 \\
\hline Thrombocytopenia & 0 & 0 & 0 & $3(13.6)$ & 0.030 \\
\hline \multicolumn{6}{|l|}{ Renal involvement } \\
\hline Proteinuria & $11(44)$ & $5(62.5)$ & $13(100)$ & $6(27.3)$ & 0.005 \\
\hline$<500 \mathrm{mg}$ & $4(16)$ & 0 & 0 & $1(4.5)$ & \\
\hline $500-1000 \mathrm{mg}$ & $10(40)$ & $3(37.5)$ & 0 & $15(68.2)$ & \\
\hline \multicolumn{6}{|l|}{$>1500 \mathrm{mg}$} \\
\hline Arthralgia/arthritis & $7(28)$ & $2(25)$ & 0 & $6(27.2)$ & 0.001 \\
\hline SLEDAI-2K at diagnosis (IQR) & $10.1(6.13)$ & $5.8(2.8)$ & $\mathrm{N} / \mathrm{A}$ & $7.2(4.11)$ & 0.296 \\
\hline
\end{tabular}

Bold indicates statistically significant

$A C L E$ acute cutaneous lupus erythematosus, $A N A$ Anti-nuclear antibody; Anti-dsDNA anti-double stranded DNA antibodies, Anti-Sm antibody Anti-Smith antibody, $C$ complement, CCLE chronic cutaneous lupus erythematosus, ESR erythrocyte sedimentation rate, SCLE subacute cutaneous lupus erythematosus, SLEDAI-2K Systemic Lupus Erythematosus Disease Activity Index

disease. Cutaneous manifestations are central to the primary diagnosis of SLE, thus complete comprehension of CLE is important to achieve optimum management and the most appropriate patient monitoring. From our study, among 101 patients diagnosed with CLE, CCLE was the most common subtype (found in 60.4\%), followed by ACLE (24.8\%) and SCLE (7.9\%). Asian and Black ethnic groups are known to have high incidence of CCLE. Our results are similar to the literature on Asian [14, 15] and Black people $[20,21]$, as well as Caucasian people $[2,9,21,22]$. Although statistically insignificant, patients with SCLE and CCLE 
were older than those with ACLE, corresponding to previous studies $[23,24]$. We found substantial differences in gender distribution between CLE subtypes, with the highest female predominance in ACLE and CCLE with SLE (female-to-male ratio of $20: 1$ and 10:1, respectively). A notable decline in the female-to-male ratio was observed in the CLE-only groups (SCLE 7:1 and CCLE 2.3:1). Female preponderance is well known in SLE, therefore as expected, CLE subtypes with concomitant SLE possessed a similarly high female distribution. However, the prevalence of CLE-only cases increased in male gender along with increasing age [25]. Hormonal factors may play an important role in the pathogenesis of SLE and CLE $[26,27]$. Androgen has been postulated to be antiinflammatory [28]. Reduction of estrogen in postmenopausal woman, and a possible protective role of androgen, may be associated with a decrease in incidence of CLE in women and increase in men with advancing age.

LE-nonspecific cutaneous manifestations commonly occur in patients who do not have SLE, thus such skin lesions are often underestimated. The present study indicates that identifying LE-nonspecific lesions is important because their presence implies systemic involvement and high disease activity. Previous studies have detected LE-nonspecific skin lesions in patients with SLE, and usually in the active phase of disease [22]. Like Vera-Recabarren et al. [29], we demonstrated that LE-nonspecific cutaneous manifestations occurred not only in SLE, but also CCLE cases without SLE. On further analysis, we found that CCLE with any LE-nonspecific lesions implied higher systemic involvement and disease activity compared with those without. The difference in the basic pathophysiologic mechanism implicated for LE-specific (T-cell-mediated immune response) and LE-nonspecific (mostly of immune complex-mediated damage) lesions may explain our result [30]. Perhaps a more complex mechanism existed in CLE patients with coexisting LE-nonspecific lesions and may have led to higher disease activity.

Histopathological examination of lesions plays a major role in diagnosis of CLE. In the present study, we found that histopathological features of SCLE, and CCLE with and without SLE demonstrated more prominent and deeper infiltration of inflammatory cells around the vessels and eccrine compared with ACLE, which is in line with the work of Oh et al. [15]. These findings correlated well with the clinical manifestations, where ACLE presented with edematous macular erythema and was biopsied early when the lesion was less established. SCLE and CCLE, on the other hand, were thicker and more developed, thereby possessing deeper infiltrations. Our findings differ to a certain extent from other reports where CCLE (classic DLE) lesions had a higher portion of epidermal atrophy, vacuolar alteration, basement membrane thickening, and follicular plugging $[14,31]$. The histopathologic spectrum of CLE can be affected by both the duration of lesion and the time of patient visit, which may have also led to the discrepancy on histopathologic results. It has been generally accepted that SCLE and CCLE share similar histopathologic findings. There are contradictory reports on distinguishing features between them [32, 33]. However, in our study, focal hypergranulosis was prominent in SCLE sections. We also suggest that pathological confirmation of CLE allows stronger data on uncommon CCLE subtypes; For example, lobular panniculitis and hyalinized sclerosis were significant findings for the CCLE-only subgroup and were found exclusively in LE panniculitis.

Lesional DIF illustrates features that may help distinguish CLE from other skin disorders. However, information on the DIF findings in relation to histopathology and systemic involvement is not well documented or studied. Our results show that DIF was positive in the majority of CLE patients. There was a borderline association between perivascular/periadnexal infiltration on histology and dermal blood vessel/periadnexal deposition on DIF $(p=0.069)$. Our sample size was likely too small to demonstrate a significant relationship between histopathology and the corresponding DIF findings. Moreover, unlike histopathology, DIF does not reflect real-time changes but displays any past insult on the structures involved. In terms of DIF features in relation to systemic involvement, CLE without SLE was the only 
subgroup with staining of solitary immunoreactant, while multiple staining was significantly associated with internal lupus. We suggest that multiple rather than single immunoreactant on lesional skin may likely imply systemic involvement. A study by Luo et al. [34] also demonstrated that patients with positive DIF had severe SLE, and $>1$ immunoreactant on lesional skin correlated to higher immunological profile and SLE disease activity. A study conducted on non-lesional skin also revealed that higher number of cutaneous immunoreactant correlated with greater disease activity [35].

In this study, we confirmed some known associations between autoantibodies and CLE subtypes. CCLE-only patients had significantly lower percentage of positive ANA, anti-dsDNA, and anti-ENA, as well as lower inflammatory and immunological profiles [36, 37]. ANA and anti-dsDNA antibodies, as per definition, were more prevalent in SLE (ACLE, CCLE with SLE) patients. Among anti-ENA, only anti-SSA was significantly found in SCLE patients. In our Asian population, we confirmed the lower number of SCLE cases compared with nonAsians, thus we did not have sufficient statistical power to identify the association between SCLE and other serology profiles such as antiSM, anti-RNP, and anti-SSB [35-37]. Elevated ANA titer has been identified as a marker of systemic involvement in CCLE patients [38]. Previous reports have shown great discrepancy on the ANA titer between CCLE with and without SLE [29, 37]. We also found a sizable difference on the ANA titer, where higher percentage of CCLE patients without SLE had lower ANA titer (71.8\%), while the majority of CCLE with SLE cases had moderate to high ANA titer (86.4\%).

Approximately half of our CLE patients had SLE at the disease onset. Our results were similar to a large European database analysis of CLE patients [9]. The nature of CLE appears to be nonstatic, as evidenced by the progression of a patient's disease to SLE through follow-up. There have been mixed reports on the percentage of patients who experience CLE-SLE transformation ranging from $5 \%$ to $23 \%[9,39,40]$. Disease translation appears to be gradual with a mean duration between CLE and SLE diagnosis of approximately 2.8 years [8]. Using the 2012 SLICC classification criteria, we demonstrated that 38 patients remained with CLE only and 4 (9.5\%) met SLE criteria during follow-up period with a median progression time of 5.6 (5.1, 44.9) months. During onset of SLE conversion, $75 \%$ of our patients went on to develop hematologic and/or renal involvement, while 25\% had only mucocutaneous signs with positive serology. However, the overall disease severity remained mild. It is challenging to make a direct comparison of our data with earlier studies because most of them used the American College of Rheumatology (ACR) criteria to establish the diagnosis of SLE. When using the ACR criteria, 4 of 11 criteria were required, including malar rash, discoid rash, photosensitivity, and oral ulcers, which are all cutaneous manifestations. Most importantly, patients with CLE who met ACR criteria for SLE did so mainly by mucocutaneous criteria [8]. As dermatologists are responsible for screening SLE in CLE patients, determining high risk characteristics is important; however, these specific profiles are not fully characterized. Our findings on the predictors for transformation showed that all were female with moderate to high ANA titer. Two had generalized DLE, two had lupus panniculitis, and all had accompanying non-scarring alopecia. Remarkably, non-scarring alopecia in SLE has been documented to hold prognostic values for SLE, and its coexistence with CLE may imply a higher risk of translation to SLE $[41,42]$. Future prospective studies with larger sample sizes are needed to confirm our findings.

The present study is subjected to several limitations. First, this was a single-center study carried out at our referral hospital, therefore the study may not represent the general population. Second, we conducted a uniform population analysis on Thai patients, and thus results may not be universally applicable to all ethnicities. Third, our observation period was relatively short, and patients could develop systemic symptoms in the future. Finally, the present analysis did not consider therapeutic management. Despite these limitations, this study could provide many benefits as it 
prospectively evaluated diverse features in terms of CLE classification in a Thai population which has never been documented before.

\section{CONCLUSION}

Our prospective cohort of Thai patients confirms that each CLE subtype has a diverse and unique character. The correlation between clinical, histopathology, immunofluorescence, and serologic profiles remains crucial for accurate diagnosis. From the clinical aspect, we emphasize the importance of thoroughly examining for LE-nonspecific lesions in CLE patients, as they are indicators of internal lupus with high disease severity and progression to SLE. Histopathologic studies showed distinctive features for each CLE subtype and contributed toward confirming the diagnosis. We demonstrated that multiple immunoreactant staining on DIF may indicate systemic disease. Approximately $10 \%$ of exclusive CLE patients can progress to SLE. Fortunately, those who progress had mild systemic disease. We anticipate that this report could clarify the distinct aspects and heterogeneity of CLE subtypes for dermatologists to embrace their role in making a correct diagnosis and providing appropriate disease monitoring.

\section{ACKNOWLEDGEMENTS}

We would like to thank the all participants in this study. We also appreciate Dr. Kunlawat Thadanipon from Department of Clinical Epidemiology and Biostatistics for statistical analysis, and also thank the Department of Pathology, Faculty of Medicine, Ramathibodi Hospital, for preparation of biopsy specimens.

Funding. This is an investigator-initiated study and no funding or sponsorship was received for this study or publication of this article.

Authorship. The named author meets the International Committee of Medical Journal Editors (ICMJE) criteria for authorship for this article, takes responsibility for the integrity of the work as a whole, and has given their approval for this version to be published.

Authorship Contributions. All writers and contributors who participated in the preparation of the manuscript are listed as authors.

Disclosures. Kumutnart Chanprapaph, Jutamas Tankunakorn, Poonkiat Suchonwanit and Suthinee Rutnin declare that they have nothing to disclose.

Compliance with Ethics Guidelines. The study protocol was approved by Mahidol University Institutional Review Board for Ethics in Human Research (MURA2560/266). All procedures performed involving human participants were in accordance with the institutional research committee and with the 1964 Helsinki Declaration and its later amendments or comparable ethical standards. Informed consent to participate in the study and for the publication of this article was obtained from all individual participants included in the study.

Data Availability. The datasets generated and analyzed in the current study are available from the corresponding author on reasonable request.

Open Access. This article is licensed under a Creative Commons Attribution-NonCommercial 4.0 International License, which permits any non-commercial use, sharing, adaptation, distribution and reproduction in any medium or format, as long as you give appropriate credit to the original author(s) and the source, provide a link to the Creative Commons licence, and indicate if changes were made. The images or other third party material in this article are included in the article's Creative Commons licence, unless indicated otherwise in a credit line to the material. If material is not included in the article's Creative Commons licence and your intended use is not permitted by statutory regulation or exceeds the permitted use, you will need to obtain permission directly from the copyright holder. To view a copy of this licence, 
visit http://creativecommons.org/licenses/bync/4.0/.

\section{REFERENCES}

1. Provost TT. The relationship between discoid and systemic lupus erythematosus. Arch Dermatol. 1994;130:1308-10.

2. Petersen MP, Möller S, Bygum A, Voss A, Bliddal M. Epidemiology of cutaneous lupus erythematosus and the associated risk of systemic lupus erythematosus: a nationwide cohort study in Denmark. Lupus. 2018;27:1424-30.

3. Petri M, Orbai AM, Alarcón GS, et al. Derivation and validation of the Systemic Lupus International Collaborating Clinics classification criteria for systemic lupus erythematosus. Arthritis Rheum. 2012;64:2677-86.

4. Hochberg MC. Updating the American College of Rheumatology revised criteria for the classification of systemic lupus erythematosus. Arthritis Rheum. 1997;40:1725.

5. Aringer M, Costenbader K, Daikh D, et al. 2019 European League Against Rheumatism/American College of Rheumatology Classification criteria for systemic lupus erythematosus. Arthritis Rheumatol. $2019 ; 71: 1400-12$.

6. Kuhn A, Landmann A. The classification and diagnosis of cutaneous lupus erythematosus. J Autoimmun. 2014;48-49:14-9.

7. Grönhagen CM, Gunnarsson I, Svenungsson E, Nyberg F. Cutaneous manifestations and serological findings in 260 patients with systemic lupus erythematosus. Lupus. 2010;19:1187-94.

8. Wieczorek IT, Propert KJ, Okawa J, Werth VP. Systemic symptoms in the progression of cutaneous to systemic lupus erythematosus. JAMA Dermatol. 2014;150:291-6.

9. Durosaro O, Davis MD, Reed KB, Rohlinger AL. Incidence of cutaneous lupus erythematosus, 1965-2005: a population-based study. Arch Dermatol. 2009;145:249-53.

10. Gilliam JN, Sontheimer RD. Distinctive cutaneous subsets in the spectrum of lupus erythematosus. J Am Acad Dermatol. 1981;4:471-5.

11. Obermoser G, Sontheimer RD, Zelger B. Overview of common, rare and atypical manifestations of cutaneous lupus erythematosus and histopathological correlates. Lupus. 2010;19:1050-70.

12. Walling HW, Sontheimer RD. Cutaneous lupus erythematosus: issues in diagnosis and treatment. Am J Clin Dermatol. 2009;10:365-81.

13. Watanabe $T$, Tsuchida $T$. Classification of lupus erythematosus based upon cutaneous manifestations. Dermatological, systemic and laboratory findings in 191 patients. Dermatology. 1995;190: 277-83.

14. Ng PP, Tan SH, Koh ET, Tan T. Epidemiology of cutaneous lupus erythematosus in a tertiary referral centre in Singapore. Australas J Dermatol. 2000;41: 229-33.

15. Oh EH, Kim EJ, Ro YS, Ko JY. Ten-year retrospective clinicohistological study of cutaneous lupus erythematosus in Korea. J Dermatol. 2018;45:436-43.

16. Mariz HA, Sato EI, Barbosa $\mathrm{SH}$, Rodrigues $\mathrm{SH}$, Dellavance A, Andrade LE. Pattern on the antinuclear antibody-HEp-2 test is a critical parameter for discriminating antinuclear antibody-positive healthy individuals and patients with autoimmune rheumatic diseases. Arthritis Rheum. 2011;63: 191-200.

17. Gladman DD, Ibañez D, Urowitz MB. Systemic lupus erythematosus disease activity index 2000. J Rheumatol. 2002;29:288-91.

18. Yee CS, Isenberg DA, Prabu A, et al. BILAG-2004 index captures systemic lupus erythematosus disease activity better than SLEDAI-2000. Ann Rheum Dis. 2008;67:873-6.

19. Isenberg DA, Rahman A, Allen E, BILAG, et al. Development and initial validation of an updated version of the British Isles Lupus Assessment Group's disease activity index for patients with systemic lupus erythematosus. Rheumatol (Oxf). 2004;2005(44):902-6.

20. Koch K, Tikly M. Spectrum of cutaneous lupus erythematosus in South Africans with systemic lupus erythematosus. Lupus. 2019;28:1021-6.

21. Drenkard C, Parker S, Aspey LD, et al. Racial disparities in the incidence of primary chronic cutaneous lupus erythematosus in the Southeastern US: the Georgia Lupus Registry. Arthritis Care Res (Hobok). 2019;71:95-103.

22. Cardinali C, Caproni M, Bernacchi E, Amato L, Fabbri P. The spectrum of cutaneous manifestations in lupus erythematosus-the Italian experience. Lupus. 2000;9:417-23. 
23. Biazar C, Sigges J, Patsinakidis N, et al. Cutaneous lupus erythematosus: first multicenter database analysis of 1002 patients from the European Society of Cutaneous Lupus Erythematosus (EUSCLE). Autoimmun Rev. 2013;12:444-54.

24. Vera-Recabarren M, Carrasco M, Cervera R, Herrero C. Comparative analysis of acute cutaneous lupus erythematosus with subacute and chronic cutaneous lupus erythematosus: clinical and immunological study of 308 patients. J Arthritis. 2016;5: 1000185 .

25. Jarukitsopa S, Hoganson DD, Crowson CS, et al. Epidemiology of systemic lupus erythematosus and cutaneous lupus erythematosus in a predominantly white population in the United States. Arthritis Care Res (Hobok). 2015;67:817-28.

26. Gulati G, Brunner HI. Environmental triggers in systemic lupus erythematosus. Semin Arthritis Rheum. 2018;47:710-7.

27. Oktem O, Yagmur H, Bengisu H, Urman B. Reproductive aspects of systemic lupus erythematosus. J Reprod Immunol. 2016;117:57-65.

28. Härle P, Pongratz G, Weidler C, Büttner R, Schölmerich J, Straub RH. Possible role of leptin in hypoandrogenicity in patients with systemic lupus erythematosus and rheumatoid arthritis. Ann Rheum Dis. 2004;63:809-16.

29. Vera-Recabarren MA, García-Carrasco M, RamosCasals M, Herrero C. Comparative analysis of subacute cutaneous lupus erythematosus and chronic cutaneous lupus erythematosus: clinical and immunological study of 270 patients. Br J Dermatol. 2010;162:91-101.

30. Zecević RD, Vojvodić D, Ristić B, Pavlović MD, Stefanović D, Karadaglić D. Skin lesions-an indicator of disease activity in systemic lupus erythematosus? Lupus. 2001;10:364-7.

31. Crowson AN, Magro C. The cutaneous pathology of lupus erythematosus: a review. J Cutan Pathol. 2001;28:1-23.

32. Jerdan MS, Hood AF, Moore GW, Callen JP. Histopathologic comparison of the subsets of lupus erythematosus. Arch Dermatol. 1990;126:52-5.

33. Bielsa I, Herrero C, Collado A, Cobos A, Palou J, Mascaró JM. Histopathologic findings in cutaneous lupus erythematosus. Arch Dermatol. 1994;130: $54-8$.

34. Luo YJ, Tan GZ, Yu M, et al. Correlation of cutaneous immunoreactants in lesional skin with the serological disorders and disease activity of systemic lupus erythematosus. PLoS ONE. 2013;8:e70983.

35. Zecević RD, Pavlović MD, Stefanović D. Lupus band test and disease activity in systemic lupus erythematosus: does it still matter? Clin Exp Dermatol. 2006;31:358-60.

36. Verdelli A, Coi A, Marzano AV, et al. Autoantibody profile and clinical patterns in 619 Italian patients with cutaneous lupus erythematosus. J Eur Acad Dermatol Venereol. 2019;33:742-52.

37. Patsinakidis N, Gambichler T, Lahner N, Moellenhoff K, Kreuter A. Cutaneous characteristics and association with antinuclear antibodies in 402 patients with different subtypes of lupus erythematosus. J Eur Acad Dermatol Venereol. 2016;30: 2097-104.

38. Chong BF, Song J, Olsen NJ. Determining risk factors for developing systemic lupus erythematosus in patients with discoid lupus erythematosus. $\mathrm{Br} \mathrm{J}$ Dermatol. 2012;166:29-35.

39. O'Brien JC, Chong BF. Not just skin deep: systemic disease involvement in patients with cutaneous lupus. J Investig Dermatol Symp Proc. 2017;18:S6974.

40. Grönhagen CM, Fored CM, Granath F, Nyberg F. Cutaneous lupus erythematosus and the association with systemic lupus erythematosus: a population-based cohort of 1088 patients in Sweden. Br J Dermatol. 2011;164:1335-41.

41. Chanprapaph K, Udompanich S, Visessiri Y, Ngamjanyaporn P, Suchonwanit P. Nonscarring alopecia in systemic lupus erythematosus: a crosssectional study with trichoscopic, histopathologic, and immunopathologic analyses. J Am Acad Dermatol. 2019;81:1319-29.

42. Suchonwanit P, Udompanich S, Thadanipon K, Chanprapaph K. Trichoscopic signs in systemic lupus erythematosus: a comparative study with 109 patients and 305 healthy controls. J Eur Acad Dermatol Venereol. 2019;33:774-80. 